\title{
Computed tomography-quantified emphysema distribution is associated with lung function decline
}

Firdaus A.A. Mohamed Hoesein*, Eva van Rikxoort", , Bram van Ginneken\#, Pim A. de Jong ${ }^{+}$, Mathias Prokop ${ }^{\S}$, Jan-Willem J. Lammers* and Pieter Zanen*

ABSTRACT: Emphysema distribution is associated with chronic obstructive pulmonary disease. It is, however, unknown whether computed tomography (CT)-quantified emphysema distribution (upper/lower lobe) is associated with lung function decline in heavy (former) smokers.

587 male participants underwent lung CT and pulmonary function testing at baseline and after a median (interquartile range) follow-up of 2.9 (2.8-3.0) yrs. The lungs were automatically segmented based on anatomically defined lung lobes. Severity of emphysema was automatically quantified per anatomical lung lobe and was expressed as the 15th percentile (Hounsfield unit point below which $15 \%$ of the low-attenuation voxels are distributed (Perc15)). The CT-quantified emphysema distribution was based on principal component analysis. Linear mixed models were used to assess the association of emphysema distribution with forced expiratory volume in $1 \mathrm{~s}$ (FEV1)/forced vital capacity (FVC), FEV1 and FVC decline.

Mean \pm SD age was $60.2 \pm 5.4$ yrs, mean baseline FEV $1 / F V C$ was $71.6 \pm 9.0 \%$ and overall mean Perc15 was $-908.5 \pm 20.9 \mathrm{HU}$. Participants with upper lobe-predominant CT-quantified emphysema had a lower FEV1/FVC, FEV1 and FVC after follow-up compared with participants with lower lobe-predominant CT-quantified emphysema $(p=0.001)$, independent of the total extent of CTquantified emphysema.

Heavy (former) smokers with upper lobe-predominant CT-quantified emphysema have a more rapid decrease in lung function than those with lower lobe-predominant CT-quantified emphysema.

KEYWORDS: Chronic obstructive pulmonary disease, computed tomography, emphysema, lung function decline, smoking

hronic obstructive pulmonary disease (COPD) is one of the major causes of morbidity and mortality worldwide [1]. COPD consists of chronic bronchitis and emphysema, which both may lead to airflow obstruction. Emphysema is defined as an abnormal and permanent enlargement of the air spaces distal to the terminal bronchioles and destruction of bronchial walls, which, in the vast majority of cases in the Western world, is caused by tobacco smoking. Although emphysema is a pathological diagnosis it may also be assessed by quantitative computed tomography $(\mathrm{CT})$ measuring low-attenuation areas (LAAs) of the lung. This technique has been validated against pathology [2] and has been used in multiple studies [3-5].
Since lung cancer and COPD share smoking as a mutual risk factor, participants of lung cancer screening trials provide the unique opportunity to study the relationships between CT-quantified emphysema and lung function decline in relatively healthy smokers [6]. The results may be useful to select participants in need of more aggressive smoking cessation therapies to prevent further lung function deterioration at a fairly early stage of the disease.

Several studies have shown that subjects with similar degrees of LAAs, but with different locations within the lung, show different degrees of airflow obstruction [7, 8]. However, those studies were cross-sectional and the effects of the

\section{AFFILIATIONS}

*Division of Heart and Lungs, Dept of Respiratory Medicine, University Medical Center Utrecht,

\#Image Sciences Institute, Dept of Radiology, University Medical Center Utrecht,

${ }^{+}$Dept of Radiology, University Medical Center Utrecht, Utrecht, "Diagnostic Image Analysis Group, Dept of Radiology, Radboud University Nijmegen Medical Center, and

${ }^{\S}$ Dept of Radiology, Radboud

University Nijmegen Medical Center, Nijmegen, The Netherlands.

\section{CORRESPONDENCE}

F.A.A. Mohamed Hoesein

Division of Heart and Lungs

Dept of Respiratory Medicine University Medical Center Utrecht Heidelberglaan 100 3508 GA Utrecht

The Netherlands

E-mail: fmohamedhoesein@ gmail.com

Received:

Oct 252012

Accepted after revision:

Jan 242012

First published online:

Feb 092012 
CT-quantified emphysema distribution on disease progression, i.e. lung function decline, were not assessed. In subjects with $\alpha_{1}$-anti-trypsin (AAT) deficiency, for instance, it was shown that emphysema distribution was associated with lung function decline [4].

Recent advances enable automatic anatomically based segmentation of the lungs allowing estimation of the extent of LAAs per lung lobe, instead of, for example, per upper or lower one-third of the lung [9].

We hypothesise that, like in AAT deficiency, distribution of LAAs in heavy smokers is associated with lung function decline. The aim of the present study was therefore to assess the effect of CT-quantified emphysema distribution, based on anatomically defined lung lobes, on lung function decline in current and former smokers participating in a lung cancer screening trial.

\section{METHODS}

\section{Participants}

The study was conducted among those current and former heavy smokers taking part in the Dutch-Belgian Lung Cancer Screening Trial (NELSON). In the current study, only participants who underwent CT and pulmonary function tests (PFTs) at the University Medical Center Utrecht (Utrecht, the Netherlands) were included. The inclusion criteria have been described in detail elsewhere [10, 11]. In brief, the NELSON study is a population-based CT screening trial for lung cancer that studies current and former heavy smokers fit enough to undergo surgery. Both the Dutch Ministry of Health and the Medical Ethics Committee of University Medical Center Utrecht approved the study protocol and informed consent was obtained from all participants. The NELSON trial is registered at www.trialregister.nl with trial number ISRCTN63545820. For this sub-study, the original approval and informed consent allowed use of data for future research. Participants meeting the inclusion criteria of $\geqslant 20$ pack-yrs of smoking exposure were invited to participate. As fewer females in the Dutch population show the same long-term exposure to cigarettes as males, only males were included. Baseline details on smoking habits were gathered through questionnaires that included questions about the duration of smoking habit, number of pack-years smoked and smoking status (current or former smoker).

\section{Pulmonary function tests}

PFTs were performed with standardised equipment according to European Respiratory Society/American Thoracic Society guidelines and included forced expiratory volume in $1 \mathrm{~s}$ (FEV1), forced vital capacity (FVC) and FEV1/FVC [12]. For demographic purposes we labelled participants with a FEV1/FVC $<70 \%$ and alternatively below the lower limit of normal as having "airflow obstruction". Bronchodilatation was not applied [13].

\section{Computed tomography}

All participants received low-dose CT with 16-detector multidetector scanners (Mx8000 IDT or Brilliance 16P; Philips Medical Systems, Cleveland, $\mathrm{OH}, \mathrm{USA})$. Scan data were obtained in spiral mode, with $16 \times 0.75-\mathrm{mm}$ collimation and in full inspiration. No spirometric gating was applied since this does not improve repeatability of lung density measurements [14, 15]. Axial images were reconstructed with $1.0-\mathrm{mm}$ thickness at $0.7-\mathrm{mm}$ increments. All scans were reconstructed with a soft reconstruction filter
(Philips B) at a $512 \times 512$ matrix. Exposure settings were $30 \mathrm{~mA} \cdot \mathrm{s}$ at 120 peak kilovoltage $(\mathrm{kVp})$ or $140 \mathrm{kVp}$, depending on the participant's weight ( $\leqslant 80$ and $>80 \mathrm{~kg}$, respectively). This lowdose CT protocol has previously been used to quantify emphysema in COPD patients and heavy smokers [6, 16-18]. The vast majority of subjects was scanned on the Brilliance 16P scanner and a very small fraction $(\sim 1 \%)$ on the Mx8000 IDT scanner, which was used a back-up scanner. We repeated the analyses with exclusion of subjects scanned on the Mx8000 IDT scanner and found no significant differences in outcome.

\section{Segmentation of lungs and lobes}

In all CT scans, the lungs and lobes were automatically segmented using previously developed and evaluated software $[9,19]$. Segmentation of the lungs was performed using an algorithm based on region growing and morphological processing. Segmentation failures, for instance in case of incomplete fissures, were automatically detected based on statistical deviations from volume and shape measurements. In the cases for which failures were detected, an algorithm based on multi-atlas registration was applied to obtain the correct result. The lung segmentation software was previously evaluated on 100 scans from the same screening and performed with accuracy similar to human observers [19]. The software further subdivided the lungs into the anatomical lobes (fig. 1). Two lobes were segmented in the left lung (upper and lower lobe) and three in the right lung (upper, middle and lower lobe). Lobe segmentation was initiated with a segmentation of the pulmonary fissures. Next, each voxel in the lung was assigned to one of the lobes based on its position inside the lung and relative to the fissures.

\section{Emphysema quantification}

Emphysema severity was computed for the entire lung and per lung lobe. The airways were excluded to ensure that only lung parenchyma was analysed [20]. Severity of CT-quantified emphysema was calculated using the 15th percentile (Perc15) technique [21-23]. Perc15 provides the Hounsfield unit point below which $15 \%$ of all voxels are distributed. The lower the Perc15 values are, i.e. the closer to $-1,000 \mathrm{HU}$, the more CTquantified emphysema is present. The use of Perc15 for emphysema quantification has been validated against pathology [24] and applied in multiple studies [4,6]. A secondary analysis was performed using \% $950 \mathrm{HU}$ as a CT-quantified emphysema severity measure, which is defined as the proportion of low density voxels below $-950 \mathrm{HU}$. The results of these analyses are reported in the supplemental files.

\section{Statistical analysis}

Mean \pm SD was calculated for normally distributed data and median (interquartile range) for non-normally distributed data. Normal distributions were checked via Q-Q plots. Unpaired ttests were used to compare means of normally distributed variables and Chi-squared tests for categorical variables. Correlations between the Perc15 values per lung lobe were assessed by Pearson's correlation.

The Perc15 value per lobe is expected to be highly correlated with that of the other lobes in the same participant, resulting in multicollinearity issues. Therefore, principal component analysis (PCA) with a varimax rotation was performed to obtain uncorrelated variables. 

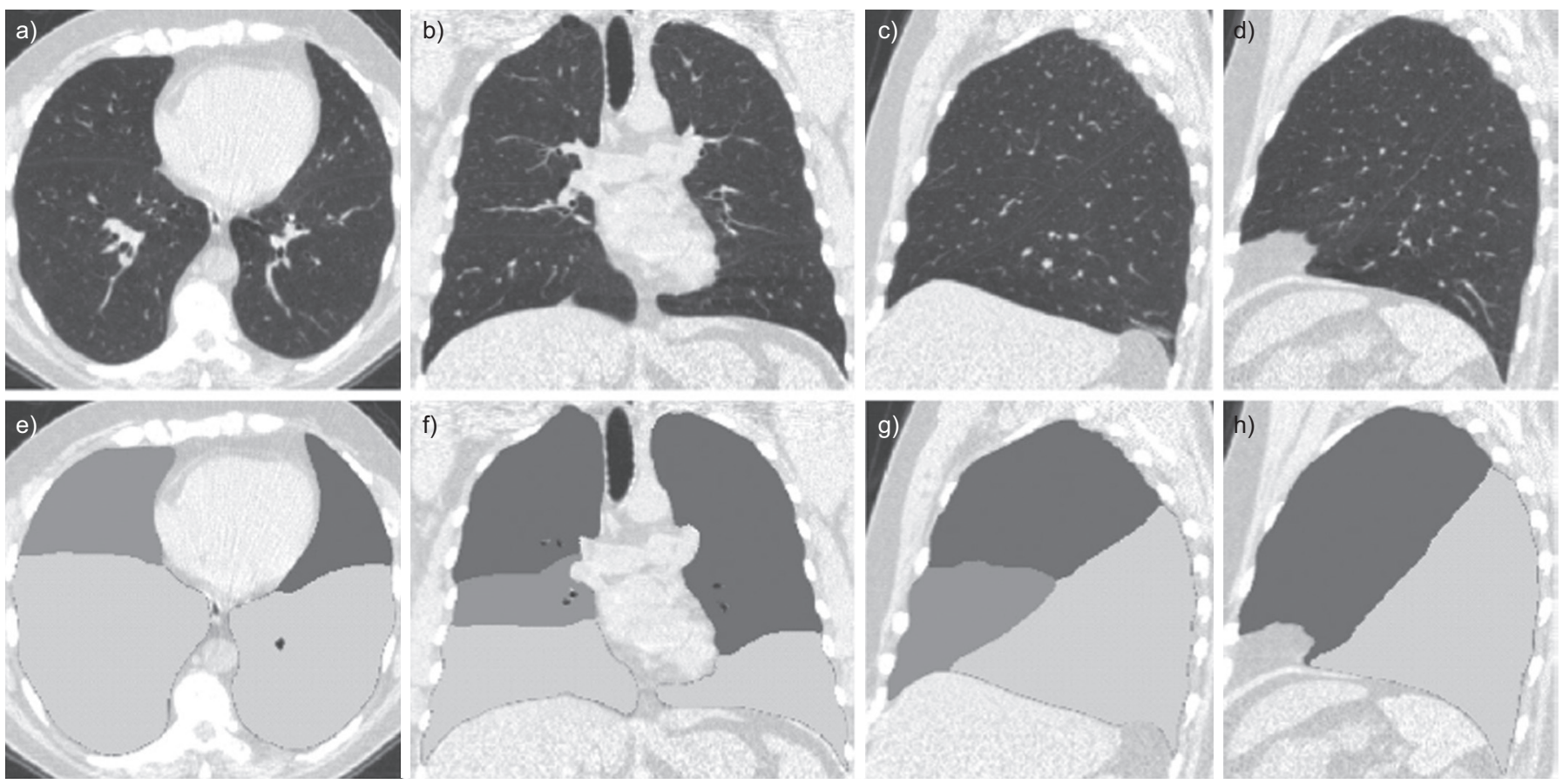

FIGURE 1. Illustration of a random a-d) computed tomography scan with e-h) the lobe segmentation as it was performed by the software. a and e) axial segmentation; $\mathrm{b}$ and f) coronal segmentation; c, d, g and h) sagittal segmentation.

PCA is a well-known data reduction technique and is often used to convert a set of correlated variables into uncorrelated ones [25]. Multicollinearity issues are so resolved and PCA has been used recently for this purpose [26, 27]. The new variables, called "components" in PCA terminology, are linear combinations of the original ones. There is a resemblance with linear regression: the combinations are based on "regression coefficients", which in PCA are called "scores" and the linear combinations are called "components". Every component is linked to a characteristic of the original set of variables and is often referred to as "phenotypes". The first component obtained is often a mean of the original variables and therefore explains the greatest proportion of variance. The second and subsequent components describe other phenotypes. The percentage of variance explained by the second component will be less than by the first component. This procedure goes on until all the variance is explained; however, each subsequent component will explain a smaller proportion. Higher components can be ignored as the percentage of additional variance explained is minimal. Only components explaining $>5 \%$ were retained in this case.

The values of the new variables (called factor scores) are subsequently incorporated in a random-intercept, random-slope linear mixed model with FEV1/FVC, FEV1 and FVC per timepoint as the primary end-point. Three separate models were created and compared. The first model contained observation time, height, body mass index (BMI), age, pack-years smoked and smoking status (i.e. being a continuous smoker or not). In a second model, component 1 values were added and, in a third model, both component 1 and 2 values. The -2 restricted loglikelihood values were used to evaluate whether insertion of more components improved the fit of the model significantly. A $\mathrm{p}$-value $\leqslant 0.05$ was considered significant. A detailed description of the statistical methods is given in the supplemental files. All statistical analyses were performed using SPSS 19 (SPSS, Chicago, IL, USA).

\section{RESULTS}

\section{Baseline demographics and lung function}

A total of 609 participants underwent baseline and follow-up CT and PFTs. After exclusion of 22 participants because the software failed to segment the lung lobes, 587 participants were included in the current study. Mean \pm SD baseline FEV1 was $97.7 \pm 18.1 \%$ predicted and FEV1/FVC was $71.6 \pm 9.0 \%$. Further baseline demographics and lung function values are presented in table 1 .

\section{Smoking status}

Mean \pm SD smoking exposure was $41.2 \pm 18.7$ pack-yrs. At enrolment of the study, 305 (50.1\%) participants were former smokers and $304(49.9 \%)$ were current smokers. The number of packyears smoked did not significantly differ between current and former smokers (38.8 and 41.1 pack-yrs, respectively; $\mathrm{p}=0.251$ ).

\section{Baseline CT-quantified emphysema: results from PCA}

Overall mean \pm SD baseline Perc15 was $-908.5 \pm 20.9$ HU. Perc15 per lung lobe is given in table 2 . The Perc15 values between the five lung lobes were highly correlated (correlation coefficient ranging from 0.75 to 0.950 , all $\mathrm{p}<0.0001$ ). Two components or phenotypes were defined, which explained $94 \%$ of the variance (component 1, 86.1\%; component 2, 7.9\%). Table 2 shows the scores of the two components. Component 1 relates to the overall mean Perc15 and is interpreted as "total lung emphysema severity" (ES). Component 2 relates to "upper/lower lobe emphysema severity" (emphysema distribution (ED)). The positive coefficients (scores) for component 2 in table 2 relate to a predominantly upper lobe-distributed emphysema and the negative coefficients to a predominantly lower lobe distribution. 


\begin{tabular}{|c|c|c|}
\hline TABLE 1 & \multicolumn{2}{|c|}{$\begin{array}{l}\text { Baseline participants' demographics for the total } \\
\text { cohort }\end{array}$} \\
\hline \multicolumn{2}{|l|}{ Subjects $n$} & 587 \\
\hline \multicolumn{2}{|l|}{ Age yrs } & $60.2 \pm 5.4$ \\
\hline \multicolumn{2}{|l|}{ Height $\mathrm{m}$} & $1.78 \pm 0.07$ \\
\hline \multicolumn{2}{|l|}{$\mathrm{BMI} \mathbf{k g} \cdot \mathrm{m}^{-2}$} & $26.9 \pm 3.6$ \\
\hline \multicolumn{2}{|c|}{ Observation time yrs median (IQR) } & $2.9(2.8-3.0)$ \\
\hline \multicolumn{2}{|c|}{ Smoking exposure pack-yrs } & $41.2 \pm 18.7$ \\
\hline \multicolumn{2}{|c|}{ Current smokers } & $304(49.9)$ \\
\hline \multicolumn{2}{|l|}{ FEV1 L } & $3.36 \pm 0.73$ \\
\hline \multicolumn{2}{|l|}{ FEV $1 \%$ pred } & $97.7 \pm 18.1$ \\
\hline \multicolumn{2}{|l|}{ FVC L } & $4.96 \pm 0.82$ \\
\hline \multicolumn{2}{|l|}{ FVC \% pred } & $107.2 \pm 15.1$ \\
\hline \multicolumn{2}{|l|}{ FEV $_{1 / F V C} \%$} & $71.6 \pm 9.0$ \\
\hline \multicolumn{2}{|c|}{$\mathrm{FEV}_{1 / \text { FVC }}<70 \%$} & $218(35.8)$ \\
\hline \multicolumn{2}{|c|}{$\mathrm{FEV}_{1} / \mathrm{FVC}<\mathrm{LLN}$} & $106(17.9)$ \\
\hline \multicolumn{2}{|c|}{ Total lung emphysema severity ${ }^{\#} \mathrm{HU}$} & $-908.5 \pm 20.9$ \\
\hline \multicolumn{3}{|c|}{$\begin{array}{l}\text { Data are presented as mean } \pm \mathrm{SD} \text { or } \mathrm{n}(\%) \text {, unless otherwise stated. BMI: body } \\
\text { mass index; IQR: interquartile range; FEV1: forced expiratory volume in } 1 \mathrm{~s} ; \% \\
\text { pred: \% predicted; FVC: forced vital capacity; LLN: lower limit of normal. } \\
\text { \#: expressed as Perc15, the Hounsfield unit point below which } 15 \% \text { of the low- } \\
\text { attenuation areas voxels are distributed (the lower the Perc } 15 \text { values are, i.e. the } \\
\text { closer to }-1,000 \mathrm{HU} \text {, the more emphysema is present). }\end{array}$} \\
\hline
\end{tabular}

The low coefficient for the right middle lobe indicates a minor influence. In subjects with increasing total lung emphysema severity, the ES factor score becomes more negative, based on the multiplication by the Perc15 value (e.g. -950 HU). In subjects with predominantly upper lobe emphysema, the ED becomes negative as the multiplication of the Perc15 value by the positive coefficient results in a negative factor score value, whereas a positive value results in subjects with predominantly lower lobe emphysema.

\section{Association of CT-quantified emphysema distribution and lung function after follow-up}

Median (interquartile range) follow-up time was 2.9 (2.83.0) yrs. The ES and ED values derived from the principal component analysis were inserted in the linear mixed model together with the other adjustment factors. The fit of the model significantly improved when ES and ED were inserted successively $(p<0.001)$. Both ES and ED were significantly $(p<0.001)$ associated with a lower FEV1/FVC after follow-up (table 3). A 1point decrement in the ES resulted in a $4.38 \%$ lower FEV1/FVC and a 1-point decrement in the ED resulted in an additional 1\% lower FEV1/FVC. This shows that lower Perc15 values, i.e. more LAAs, and an upper lobe predominant CT-quantified emphysema distribution are independently associated with significantly lower FEV1/FVC values after follow-up. The effects of ES and ED on FEV1/FVC after follow-up are illustrated in figure 2. ES and ED were also significantly associated with lower FEV1 and FVC after follow-up ( $<<0.001)$. The effect sizes for FEV1 and FVC are given in table 3 . The effect sizes of the other covariates in the model are also presented in table 3.

Using the \% $950 \mathrm{HU}$ approach as measure of CT-quantified emphysema severity yielded comparable results to using the Perc15 (online supplementary material).

\section{DISCUSSION}

In the present study, we showed that upper lobe distribution of CT-quantified emphysema is associated with a lower lung function after follow-up in a large cohort of 587 former and current heavy smokers participating in a lung cancer screening trial. Therefore, knowledge of the distribution of CT-quantified emphysema is important with regards to the course of lung function in former and current heavy smokers.

We used PCA to solve the problem of the high correlation between the Perc15 values per lung lobe within an individual. This approach delivered two new variables (components 1 and 2). Component 1 (ES) characterised the total extent of CTquantified emphysema, while component 2 (ED) characterised the difference between upper and lower lobe CT-quantified emphysema. The effect size of the emphysema distribution pattern $(-1.00 \%)$ is substantially higher compared with the expected normal decline of FEV1/FVC (-0.18\%) in age-matched healthy individuals. This shows that the results are of clinical importance. Ignoring the individual distribution of CT-quantified emphysema provides a less precise estimation of lung function decline.

Table 3 lists the ED and ES regression coefficients for the decline in FEV1/FVC, FEV1 and FVC, which are the results of three separate analyses. In other words the regression coefficients for

TABLE 2 15th percentile value (Perc15) per lung lobe and component scores

\section{Left upper lobe}

Right upper lobe

Right middle lobe

Left lower lobe

Right lower lobe

Variance explained \%
$-912.1+21.2$

$-906.6 \pm 23.0$

$-915.5 \pm 18.5$

$-900.5 \pm 24.7$

$-899.9 \pm 24.2$
0.218

0.212

0.214

0.216

0.217

86.15
0.743

0.937

$-0.234$

$-0.680$

$-0.759$

7.93

The two components explained $94 \%$ of the variance. Component 1 (total lung emphysema severity (ES)) is characterised by similar scores for each lobe so each lobe is equally contributing to this component; component 2 (emphysema distribution (ED)) is linked to the difference between upper and lower lobe emphysema. The component scores can be interpreted in a similar way as the regression coefficients from multiple linear regression analysis. 
TABLE 3 Results of the linear mixed models analysis

\begin{tabular}{|c|c|c|c|c|}
\hline Parameter & Unit change in parameter & Effect on FEV $1 / F V C$ & Effect on FEV 1 & Effect on FVC \\
\hline Age yrs & Increment: $1 \mathrm{yr}$ & $-0.15(-0.27--0.03)^{*}$ & $-36(-45--27)^{\star \star \star}$ & $-44(-54--35)^{\star \star \star}$ \\
\hline Height $\mathrm{cm}$ & Increment: $1 \mathrm{~cm}$ & $0.03(-0.12--0.07)^{\#}$ & $39(32-47)^{\star \star \star}$ & $55(47-63)^{\star \star \star}$ \\
\hline BMI $\mathbf{k g} \cdot \mathrm{m}^{-2}$ & Increment: $1 \mathrm{~kg} \cdot \mathrm{m}^{-2}$ & $0.18(0.02--0.38)^{\#}$ & $4.6(-11--20)^{\#}$ & $-8(-25--7)^{\#}$ \\
\hline Observation time yrs & Increment: $1 \mathrm{yr}$ & $-0.58(-0.71--0.45)^{* * *}$ & $-74(-81--67)^{\star \star *}$ & $-20(-33--6)^{* *}$ \\
\hline Smoking exposure pack-yrs & Increment: 1 pack-yr & $-0.05(-0.08--0.01)^{\star *}$ & $-5(-8--3)^{\star * *}$ & $-4(-7--1)^{\star *}$ \\
\hline ED & Decrement: 1 point & $-1.00(-0.37--1.64)^{\star *}$ & $-13(-33--2)^{*}$ & $-50(-90--10)^{*}$ \\
\hline
\end{tabular}

Data are presented as $\beta(95 \% \mathrm{Cl})$ of a unit change in each of the listed parameters. For example, the forced expiratory volume in $1 \mathrm{~s}(\mathrm{FEV} 1) / f \circ \mathrm{foced}$ vital capacity $(\mathrm{FVC})$ at a time point can be calculated using the general equation FEV1/FVC $=\alpha+\left(x_{1} \times \beta_{1}\right)+\left(x_{2} \times \beta_{2}\right)+\left(x_{3} \times \beta_{3}\right)+\ldots+\left(x_{i} \times \beta_{i}\right)$ and this results in the equation $\mathrm{FEV} 1 /$ $\mathrm{FVC}=75+($ age $\times-0.15)+($ height $\times 0.03)+(\mathrm{BMI} \times 0.18)+$ (observation time $\times-0.58)+($ smoking exposure $\times-0.05)-3.68$ (if continuous smoker) $+($ emphysema score $\times-4.38)+($ distribution score $\times-1.00)$. BMl: body mass index; ES: total lung emphysema severity (component 1); ED: emphysema distribution (component 2) *: nonsignificant $(p>0.05) .{ }^{*}: p<0.05 ;{ }^{* *}: p<0.01 ; * *: p<0.001$

FEV1/FVC decline were not obtained by simply dividing the regression coefficients of FEV1 by those of the FVC. If one is tempted to such a straightforward division, one could draw the conclusion that the FEV1/FVC ratio would increase when emphysema severity increases and that when the FEV1/FVC ratio decreases this is due to a decrease in FVC larger than the FEV1. This is obviously not the case as the ED and ES coefficients

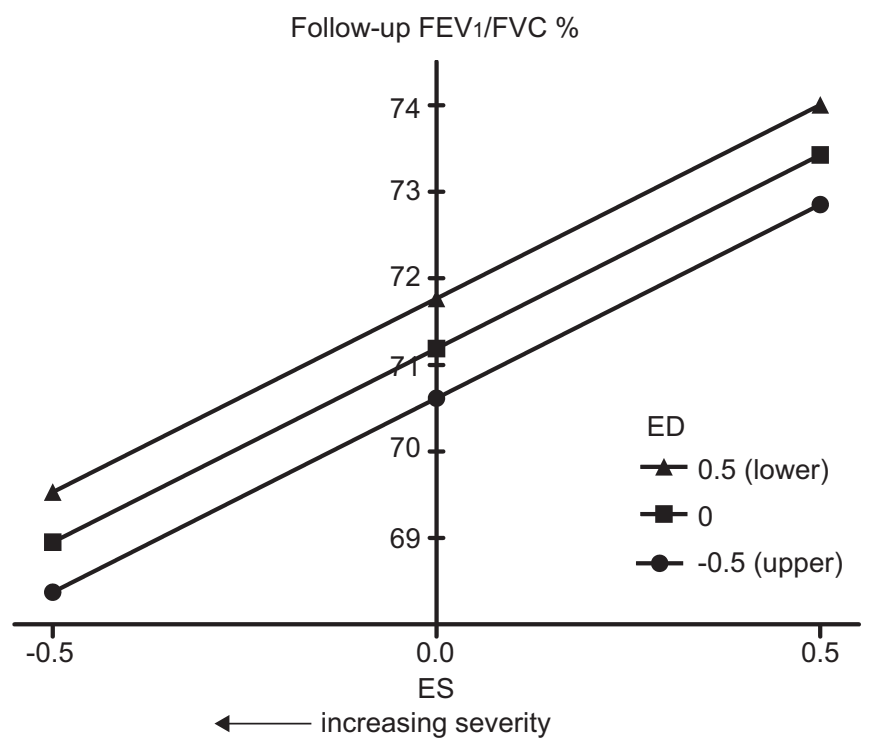

FIGURE 2. Forced expiratory volume in $1 \mathrm{~s}$ (FEV1)/forced vital capacity (FVC) ratio after 3-yr follow-up in a participant with a starting age of $60 \mathrm{yrs}$, height of $1.78 \mathrm{~m}$ and smoking exposure of 41 pack-yrs, being the mean values of the cohort. The total lung emphysema severity (ES) is depicted on the $x$-axis and the FEV1/FVC after 3 yrs on the $y$-axis. It can be seen that a lower ES value results in a lower FEV $1 /$ FVC after follow-up. The graph is stratified by the value of the emphysema distribution (ED): $-0.5,0$ and 0.5 being the first, second and third quartile, respectively. It shows that a lower ED value results in a lower FEV1/FVC after followup. For instance, an individual with upper lobe emphysema (circles) has a lower FEV1/FVC after follow-up than the individual with lower lobe emphysema (squares) despite having a similar emphysema score ( $x$-axis). for the FEV1/FVC clearly point to a decrease. One must not forget that the ED and ES are just two of the many parameters influencing the FEV1/FVC, FEV1 and FVC decline. It is not possible to isolate just the FEV1 and FVC ED and ES coefficients to obtain the FEV1/FVC coefficients. A more detailed explanation is given in the online supplementary material.

The present longitudinal study is an extension from the previous performed cross-sectional study investigating the association between emphysema distribution and lung function [8]. However, two important methodological differences exist. First, in the current study, no distinction was made between mild and severe emphysema, instead the Perc15 was used as a continuous measure for emphysema severity. We preferred this measure because it was shown to be the most robust measure for the progression of LAAs [5]. Secondly, in the previous study, segmentation of the lungs was based on a division of the lung based on volumes: upper one-third and lower one-third. Other studies also used such an approach to separate upper from lower lung fields, for instance by dividing the total lung volume in two parts [28]. Inevitably, parts of the anatomical lower lobe will be allocated to the upper lobe and our lobe segmentation, based on anatomical information, avoids this problem. Therefore, in the present study, threedimensional data and the natural boundaries of the lung were used, which enabled a reliable separation of the upper lung lobes from the lower lung lobes. Furthermore, the software used has shown to have accuracy comparable to that of an independent human observer [19].

In AAT deficiency subjects, longitudinal studies have been performed showing that lower lobe-predominant emphysema was associated with a greater decline in lung function [4]. In COPD patients without AAT deficiency, only cross-sectional studies have been performed, investigating the association of emphysema distribution with lung function. GuRNEY et al. [29] found that a lower lobe-predominant emphysema distribution was associated with lower total lung capacity values; however, these associations were only significant for subjectively quantified emphysema and not when objectively quantified. Three studies are of special interest because they quantified 
emphysema automatically, based on the percentage of LAAs below a specified threshold. SAITOH et al. [30] reported that FEV1/ FVC showed the strongest correlation with lower lobe emphysema distribution, but that the transfer factor of the lung for carbon monoxide showed the strongest correlation with upper lobe emphysema distribution. MAIR et al. [28] showed that upper zone distribution of emphysema in COPD subjects was associated with a higher total score on the St George's Respiratory Questionnaire. A higher score on the St George's Respiratory Questionnaire indicates more severe respiratory impairment. DE TORRES et al. [31] failed to find a correlation between the distribution of emphysema and lung function parameters in subjects with mild-to-moderate COPD. It was concluded that in mild COPD, emphysema distribution is not associated with lung function. However, it should be taken in account that the sample sizes might be too small $(n=115)$ to allow sufficient power for detecting true associations. Furthermore, in all studies, classification of the emphysema distribution was not anatomically based, which might have influenced the findings.

Less is known about why smokers differ in emphysema distribution pattern. Like in AAT deficiency, a genetic susceptibility may play a role $[32,33]$. Candidate gene studies in the National Emphysema Treatment Trial showed that upper lobepredominant emphysema was associated with polymorphisms in two enzymes playing a role in the detoxification of smoke metabolites [32]. The authors posed that these polymorphisms alter the normal detoxification of cigarette metabolites contributing to the distribution of emphysema. Future genomewide association studies may further elucidate the association between genetic susceptibility and emphysema distribution in heavy smokers.

In heavy smokers, upper lobe emphysema distribution is more common than lower lobe distribution (based on anatomically defined lung lobes). This is in line with the fact that emphysema in heavy smokers is mainly of the centrilobular type, which is more common in the upper lobes. It has been hypothesised that this emphysema distribution is caused by the difference in the ventilation/perfusion ratio between the upper and lower lobes [34].

There are a number of strengths to our study. First, we included a large number of participants and, therefore, could extensively correct for confounding factors, such as age, BMI, smoking status, pack-years, etc., unlike most other studies. Secondly, we included relatively healthy but heavy smoking subjects at a high risk for developing airflow obstruction. Most previous studies examining the effects of emphysema distribution included subjects with more severe COPD only. Thirdly, all CT was performed in a single centre, excluding possible scanner bias due to different algorithms used by different types of CT scanner. Finally, we used anatomically defined borders to segment the lungs, which might be more accurate than using lung volumes to segment the lungs or by a visual assessment. Visual assessment has been reported to be less reliable than when determined automatically [35]. Furthermore, the severity of CT-quantified emphysema was calculated automatically, which eliminates intra-observer variability between different readers of CT scans.

The main limitation of our current study is that only males were included, which is especially unfortunate because the prevalence of COPD in females is rising. Our results may not be extrapolated directly to females because it is known that males have more CT-quantified emphysema [17] and that sex is independently associated with upper or lower lung-predominant emphysema patterns [28]. Future studies should also include females to examine the association of emphysema distribution and lung function decline. Furthermore, as we included relatively healthy, but heavy smoking, participants and participants with COPD in Global Initiative for Chronic Obstructive Lung Disease stage I, the results may not be straightforwardly extrapolated to more severe COPD participants.

In conclusion, the distribution of CT-quantified emphysema is an additional parameter, besides the total extent of CTquantified emphysema, in predicting lung function decline. Upper lobe-predominant emphysema is significantly associated with stronger lung function decline compared to lower lobe predominance emphysema in former and current heavy smokers. These findings may be of importance because they may be useful to identify subjects with greater declines in lung function and probably eligible for more intensive smoking cessation counselling.

\section{SUPPORT STATEMENT}

Funding was provided by a European Union Seventh Framework Package grant, COPACETIC (grant number 201379).

\section{CLINICAL TRIAL}

The NELSON trial is registered at www.trialregister.nl with trial number ISRCTN63545820.

\section{STATEMENT OF INTEREST}

None declared.

\section{REFERENCES}

1 Murray CJ, Lopez AD. Alternative projections of mortality and disability by cause 1990-2020: Global Burden of Disease Study. Lancet 1997; 349: 1498-1504.

2 Gould GA, MacNee W, McLean A, et al. CT measurements of lung density in life can quantitate distal airspace enlargement-an essential defining feature of human emphysema. Am Rev Respir Dis 1988; 137: 380-392.

3 Gould GA, Redpath AT, Ryan M, et al. Lung CT density correlates with measurements of airflow limitation and the diffusing capacity. Eur Respir J 1991; 4: 141-146.

4 Parr DG, Stoel BC, Stolk J, et al. Validation of computed tomographic lung densitometry for monitoring emphysema in $\alpha_{1}$-antitrypsin deficiency. Thorax 2006; 61: 485-90.

5 Parr DG, Sevenoaks M, Deng C, et al. Detection of emphysema progression in alpha 1-antitrypsin deficiency using CT densitometry; methodological advances. Respir Res 2008; 9: 21.

6 Mohamed Hoesein FA, de Hoop B, Zanen P, et al. CT-quantified emphysema in male heavy smokers: association with lung function decline. Thorax 2011; 66: 782-787.

7 Parr DG, Stoel BC, Stolk J, et al. Pattern of emphysema distribution in $\alpha_{1}$-antitrypsin deficiency influences lung function impairment. Am J Respir Crit Care Med 2004; 170: 1172-1178.

8 Gietema HA, Zanen P, Schilham A, et al. Distribution of emphysema in heavy smokers: impact on pulmonary function. Respir Med 2010; 104: 76-82.

9 van Rikxoort EM, de Hoop B, Viergever MA, et al. Automatic lung segmentation from thoracic computed tomography scans using a 
hybrid approach with error detection. Med Phys 2009; 36: 2934-2947.

10 van Iersel CA, de Koning HJ, Draisma G, et al. Risk-based selection from the general population in a screening trial: selection criteria, recruitment and power for the Dutch-Belgian randomised lung cancer multi-slice CT screening trial (NELSON). Int J Cancer 2007; 120: 868-74.

11 van Klaveren RJ, Oudkerk M, Prokop $M$, et al. Management of lung nodules detected by volume CT scanning. N Engl J Med 2009; 361: 2221-2229.

12 Miller MR, Crapo R, Hankinson J, et al. General considerations for lung function testing. Eur Respir J 2005; 26: 153-161.

13 Hunninghake GM, Cho MH, Tesfaigzi $\mathrm{Y}$, et al. MMP12, lung function, and COPD in high-risk populations. N Engl J Med 2009; 361: 2599-2608.

14 Gierada DS, Yusen RD, Pilgram TK, et al. Repeatability of quantitative CT indexes of emphysema in patients evaluated for lung volume reduction surgery. Radiology 2001; 220: 448-454.

15 Newell JD Jr, Hogg JC, Snider GL. Report of a workshop: quantitative computed tomography scanning in longitudinal studies of emphysema. Eur Respir J 2004; 23: 769-775.

16 Shaker SB, Maltbaek N, Brand P, et al. Quantitative computed tomography and aerosol morphometry in COPD and $\alpha_{1}$-antitrypsin deficiency. Eur Respir J 2005; 25: 23-30.

17 Sverzellati N, Calabro E, Randi G, et al. Sex differences in emphysema phenotype in smokers without airflow obstruction. Eur Respir J 2009; 33: 1320-1328.

18 Gietema HA, Schilham AM, van Ginneken B, et al. Monitoring of smoking-induced emphysema with $\mathrm{CT}$ in a lung cancer screening setting: detection of real increase in extent of emphysema. Radiology 2007; 244: 890-897.

19 van Rikxoort EM, de Hoop B, van de Vorst S, et al. Automatic segmentation of pulmonary segments from volumetric chest $\mathrm{CT}$ scans. IEEE Trans Med Imaging 2009; 28: 621-630.

20 van Rikxoort EM, Prokop M, de Hoop B, et al. Automatic segmentation of the pulmonary lobes from fissures, airways, and lung borders: evaluation of robustness against missing data. Med Image Comput Comput Assist Interv 2009; 12: 263-271.

21 Coxson HO. Quantitative chest tomography in COPD research: chairman's summary. Proc Am Thorac Soc 2008; 5: 874-877.

22 Coxson HO. Quantitative computed tomography assessment of airway wall dimensions: current status and potential applications for phenotyping chronic obstructive pulmonary disease. Proc Am Thorac Soc 2008; 5: 940-945.

23 Newell JD Jr, Hogg JC, Snider GL. Report of a workshop: quantitative computed tomography scanning in longitudinal studies of emphysema. Eur Respir J 2004; 23: 769-775.

24 Gould GA, MacNee W, McLean A, et al. CT measurements of lung density in life can quantitate distal airspace enlargement-an essential defining feature of human emphysema. Am Rev Respir Dis 1988; 137: 380-392.

25 Joliffe IT, Morgan BJ. Principal component analysis and exploratory factor analysis. Stat Methods Med Res 1992; 1: 69-95.

26 Shaheen SO, Jameson KA, Syddall HE, et al. The relationship of dietary patterns with adult lung function and COPD. Eur Respir J 2010; 36: 277-284.

27 Burgel PR, Paillasseur JL, Caillaud D, et al. Clinical COPD phenotypes: a novel approach using principal component and cluster analyses. Eur Respir J 2010; 36: 531-539.

28 Mair G, Miller JJ, McAllister D, et al. Computed tomographic emphysema distribution: relationship to clinical features in a cohort of smokers. Eur Respir J 2009; 33: 536-542.

29 Gurney JW, Jones KK, Robbins RA, et al. Regional distribution of emphysema: correlation of high-resolution CT with pulmonary function tests in unselected smokers. Radiology 1992; 183: 457-463.

30 Saitoh $\mathrm{T}$, Koba $\mathrm{H}$, Shijubo $\mathrm{N}$, et al. Lobar distribution of emphysema in computed tomographic densitometric analysis. Invest Radiol 2000; 35: 235-243.

31 de Torres JP, Bastarrika G, Zagaceta J, et al. Emphysema presence, severity, and distribution has little impact on the clinical presentation of a cohort of patients with mild to moderate COPD. Chest 2011; 139: 36-42.

32 Demeo DL, Hersh CP, Hoffman EA, et al. Genetic determinants of emphysema distribution in the national emphysema treatment trial. Am J Respir Crit Care Med 2007; 176: 42-48.

33 Ito I, Nagai S, Handa T, et al. Matrix metalloproteinase-9 promoter polymorphism associated with upper lung dominant emphysema. Am J Respir Crit Care Med 2005; 172: 1378-1382.

34 Cockcroft DW, Horne SL. Localization of emphysema within the lung. An hypothesis based upon ventilation/perfusion relationships. Chest 1982; 82: 483-487.

35 Hersh CP, Washko GR, Jacobson FL, et al. Interobserver variability in the determination of upper lobe-predominant emphysema. Chest 2007; 131: 424-431. 